\title{
Organização da produção, do manejo e da comercialização de produtos apícolas: um
}

\section{foco nas ações coletivas}

\author{
Organization of the production, management and marketing of apicultural products: a focus on \\ collective actions
}

Organización de la producción, gestión y comercialización de productos apícolas: un enfoque en las acciones colectivas

\section{Resumo}

O objetivo foi abordar, na percepção dos cooperados que participaram da pesquisa, os principais aspectos envolvidos na organização da produção, manejo e comercialização de produtos apícolas, enfocando a importância da Cooperativa de Apicultores. A pesquisa foi realizada com $66 \%$ do total de apicultores cooperados. Realizou-se um levantamento dos dados primários junto aos apicultores. Para isso utilizou-se o método de levantamento através de formulário Estruturado e Não-disfarçado. Observou-se que menos da metade dos apicultores realizam a atividade apícola em terreno próprio. O produto com destaque na produção apícola do Vale do Jequitinhonha foi o mel. $33 \%$ dos apicultores utilizam uma área de $1 \mathrm{a} 20$ ha para a apicultura e apenas 14\% dos entrevistados abordam que a apicultura é a única fonte de renda. Dentre os apicultores entrevistados, 67\% são cooperados por pelo menos há 10 anos. No que tange a comercialização dos produtos apícolas, foi observada que $43 \%$ de apicultores comercializam total ou parcialmente os seus produtos sem o intermédio da cooperativa. Muitos dos apicultores entrevistados relataram ausência da infraestrutura adequada para coleta e manejo do mel. Nesse sentido a presença de uma cooperativa pode ser considerada uma vantagem, por oferecer aos apicultores associados uma infraestrutura adequada para a coleta do mel. Na opinião de $71 \%$ dos apicultores entrevistados a atuação da cooperativa é classificada como boa ou muito boa. Conclui-se que estratégias focadas em ações coletivas precisam ser priorizadas para consolidar os elos entre os envolvidos e proporcionar a condição necessária para o fortalecimento da apicultura regional.

Palavras-chave: Setor apícola; Cooperativismo; Cadeia produtiva; Vale do Jequitinhonha.

\section{Abstract}

The objective was to address, in the perception of the cooperative members who participated in the research, the main aspects involved in the organization of production, management and marketing of bee products, focusing on the importance of the Beekeepers Cooperative. The survey was conducted with $66 \%$ of the total cooperative beekeepers. A survey of primary data with beekeepers was carried out. For this, the survey method was used through a Structured 
and Undisguised form. It was observed that less than half of beekeepers carry out the beekeeping activity on their own land. The product that stood out in the beekeeping production of Vale do Jequitinhonha was honey. $33 \%$ of beekeepers use an area of 1 to 20 ha for beekeeping and only $14 \%$ of respondents mention that beekeeping is the only source of income. Among the interviewed beekeepers, $67 \%$ have been cooperated for at least 10 years. Regarding the commercialization of bee products, it was observed that $43 \%$ of beekeepers market their products totally or partially without the intermediary of the cooperative. Many of the beekeepers interviewed reported a lack of adequate infrastructure for collecting and managing honey. In this sense, the presence of a cooperative can be considered an advantage, as it offers associated beekeepers an adequate infrastructure for collecting honey. In the opinion of $71 \%$ of the interviewed beekeepers, the cooperative's performance is classified as good or very good. It is concluded that strategies focused on collective actions need to be prioritized to consolidate the links between those involved and provide the necessary condition for the strengthening of regional beekeeping.

Keywords: Beekeeping sector; Cooperativism; Production chain; Vale do Jequitinhonha.

\section{Resumen}

El objetivo fue abordar, en la percepción de los cooperativistas que participaron en la investigación, los principales aspectos involucrados en la organización de la producción, manejo y comercialización de productos apícolas, enfocándose en la importancia de la Cooperativa de Apicultores. La encuesta se realizó con el 66\% del total de apicultores cooperativos. Se realizó una encuesta de datos primarios con apicultores. Para ello, se utilizó el método de encuesta a través de un formulario Estructurado y No Disimulado. Se observó que menos de la mitad de los apicultores realizan la actividad apícola en su propia tierra. El producto que se destacó en la producción apícola de Vale do Jequitinhonha fue la miel. El 33\% de los apicultores utilizan un área de 1 a 20 ha para la apicultura y solo el $14 \%$ de los encuestados menciona que la apicultura es la única fuente de ingresos. Entre los apicultores entrevistados, el $67 \%$ ha cooperado durante al menos 10 años. En cuanto a la comercialización de productos apícolas, se observó que el $43 \%$ de los apicultores comercializan sus productos total o parcialmente sin la intermediación de la cooperativa. Muchos de los apicultores entrevistados informaron de la falta de infraestructura adecuada para recolectar y manejar la miel. En este sentido, la presencia de una cooperativa se puede considerar una ventaja, ya que ofrece a los apicultores asociados una infraestructura adecuada para la recolección de miel. En opinión del 71\% de los apicultores entrevistados, el desempeño de la cooperativa se cataloga como bueno o muy bueno. Se concluye que es necesario priorizar estrategias enfocadas en acciones colectivas para consolidar los vínculos entre los involucrados y brindar la condición necesaria para el fortalecimiento de la apicultura regional.

Palabras clave: Sector apícola; Cooperativismo; Cadena productiva; Vale do Jequitinhonha.

\section{Introdução}

A apicultura é o termo que designa a atividade que envolve o manejo de abelhas do mel em colmeias para polinização, produção de mel e outros produtos apícolas, bem como, produção de enxames e de rainhas (Fao, 2018). A atividade apícola é realizada, principalmente por agricultores que têm na criação de abelhas uma atividade complementar de renda em suas propriedades (Lengler et al., 2007). A literatura relata que a apicultura está ligada à sustentabilidade e contribuiu para a inserção de pequenos agricultores familiares na produção, contribuindo para a redução do êxodo rural, redução do desmatamento e incentivo ao reflorestamento, além da geração de empregos (Sachs, 2009). Cabe ressaltar que a apicultura não se restringe somente ao pequeno e médio produtor, uma vez que ela vem ganhando destaque no agronegócio brasileiro (Neto et al., 2020).

O cooperativismo é uma estratégia de organização onde a propriedade, a gestão e a repartição se dão de maneira cooperativa. Em meio à realidade cada vez mais globalizada, o movimento cooperativo, vem demonstrando uma importância no que se refere à produção, organização e comercialização da produção na agricultura familiar, além de ter como componente a produção de alimentos para garantir a segurança alimentar (Oliveira, 2018). Assim, as organizações de natureza coletiva podem transformar, por meio da união e cooperação, os pequenos agricultores dispersos em uma força coesa, principalmente nas relações políticas e econômicas, especialmente nas esferas da comercialização (de insumos e produtos), do processamento primário, da prestação de serviços, etc. (Lengleret al., 2007).

Oliveira (2018) ainda reforça que o cooperativismo é uma atividade que inclui os atores sociais em torno dos seus interesses comuns, apresentando-se como uma estratégia de geração de trabalho e renda, em um modelo econômico de natureza excludente. Percebe-se o quanto uma cooperativa pode transformar a realidade de muitos agricultores, em especial 
dos apicultores do Vale do Jequitinhonha-MG. Tornando-se um importante meio de fortalecimento e expansão da produção apícola, visto que a região dispõe de diversidade de flora com potencialidade na apicultura.

Diante do exposto, este estudo objetivou abordar, na percepção dos cooperados que participaram da pesquisa, os principais aspectos envolvidos na organização da produção, do manejo e da comercialização de produtos apícolas, enfocando a importância da Cooperativa de Apicultores do Vale do Jequitinhonha - COOAPIVAJE neste contexto.

\section{Metodologia}

\subsection{Caracterização da área de estudo}

O Vale do Jequitinhonha está localizado no nordeste do estado de Minas Gerais e ocupa uma área de $85 \mathrm{mil} \mathrm{km}^{2}$, possui uma população de aproximadamente 980 mil habitantes no qual mais de dois terços são residentes na zona rural (IBGE, 2010, 2015). A região tem cerca de 50 municípios e é dividida em três microrregiões: Baixo, Médio e Alto Jequitinhonha. Dispõe da Bacia hidrográfica do Rio Jequitinhonha, cujo principal afluente é o Rio Araçuaí e o clima faz parte do semiárido brasileiro, com variações ao longo de seu território. Nas microrregiões que compõem o Vale do Jequitinhonha possuem vegetação característica, sendo mata atlântica, cerrado e caatinga. O Vale possui uma grande extensão territorial e se destaca pela presença de campos rupestres, cerrados, caatinga e mata atlântica. Apresenta grande diversidade cultural, social e econômica, na qual as características diferem conforme cada microrregião (Fávero \& Monteiro, 2014).

A Cooperativa de Apicultores do Vale do Jequitinhonha - COOAPIVAJE está situada no município de Turmalina, no Baixo Jequitinhonha. Fundada em 2008, a cooperativa visa fortalecer a atividade apícola no Alto e Médio Jequitinhonha, de modo a garantir uma fonte de renda para os apicultores familiares da região. A cooperativa dispõe de mais de 30 associados, na qual auxilia no processamento e envasamento para comercialização do Mel Jequitinhonha (Minas Gerais, 2020).

\subsection{Método de pesquisa e análise de dados}

Para realização desta pesquisa, utilizou-se o levantamento dos dados primários junto aos apicultores cadastrados na COOAPIVAJE - Cooperativa de Apicultores do Vale do Jequitinhonha. Este estudo foi executado conforme os preceitos éticos de pesquisa em seres humanos (CAAE: 98183718.0.0000.5146, aprovada com Número do Parecer: 3.037.374).

$\mathrm{Na}$ execução da pesquisa de campo utilizou-se o método de levantamento através de formulário estruturado e nãodisfarçado. Este tipo de composição do formulário de pesquisa apresenta uma lista formal de perguntas a serem feitas e o objetivo da entrevista está totalmente explícito para o entrevistado (Campomar, 1981; Boyd \& Westfall, 1982).

Os dados primários obtidos com o levantamento foram:

- Informações gerais: nome, telefone, município e comunidade, tipo e tamanho do terreno, etc.

- Informações relacionadas à atividade apícola: principais produtos, produção média, tempo em que pratica apicultura, quantidade de pessoas envolvidas na atividade, se a apicultura é a única fonte de renda, etc.

- Informações relacionadas à cooperativa: tempo em que faz parte da cooperativa, se entrega o mel no entreposto, se considera a atuação da cooperativa importante, etc.

Para aplicação do formulário de pesquisa foram realizadas visitas aos apicultores que se dispuseram a colaborar com a pesquisa.

Entre os 32 apicultores cadastrados, 21 foram entrevistados, representando $66 \%$ do total de apicultores que fazem parte da cooperativa.

Para elaboração do perfil do apicultor e da cooperativa foram utilizadas as seguintes variáveis (Quadro 1): 
Research, Society and Development, v. 10, n. 10, e295101018891, 2021

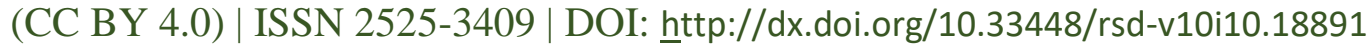

Quadro 1. Variáveis utilizadas na elaboração do perfil do apicultor e da cooperativa.

\begin{tabular}{|c|l|}
\hline Sequência & \\
\hline A & Tipo de terreno onde ficam as colmeias \\
\hline B & Tipos de produtos \\
\hline C & Área total da propriedade (ha) \\
\hline D & Área utilizada para apicultura (ha) \\
\hline E & Produção anual média de mel (kg) \\
\hline F & Tempo que pratica a apicultura (anos)? \\
\hline G & Quantidade de pessoas na atividade? \\
\hline H & Única fonte de renda? \\
\hline I & Há quanto tempo faz parte da COOAPIVAJE? (anos) \\
\hline J & Entrega a produção no entreposto? \\
\hline K & Possui casa do mel? \\
\hline L & Avaliação do trabalho da cooperativa \\
\hline
\end{tabular}

Fonte: Autores (2021).

\section{Resultados e Discussão}

Dos 32 apicultores cadastrados na Cooperativa de Apicultores do Vale do Jequitinhonha - COOAPIVAJE, 21 foram entrevistados, representando $66 \%$ do total de apicultores.

Nas análises das frequências, observou-se que menos da metade dos apicultores realizam a atividade apícola em terreno próprio e poucos utilizam parcerias ou terreno da União para o desenvolvimento da atividade. Com uma frequência de 48\% aparecem os apicultores que utilizam o terreno arrendado ou concedido para a apicultura (Figura 1A). No estudo conduzido por Gonçalves et al. (2019), na mesma região, observou-se a utilização de pasto apícola dentro de área de reflorestamento de eucalipto e que alguns apicultores pagam algum tipo de pasto apícola para conseguirem uma boa produção de mel. 
Figura 1. Dados referentes à área da propriedade e a área destinada à produção apícola. A - Tipo de terreno onde ficam as colmeias. B - Tipos de produtos. C - Área total da propriedade (ha). D - Área utilizada para apicultura (ha).

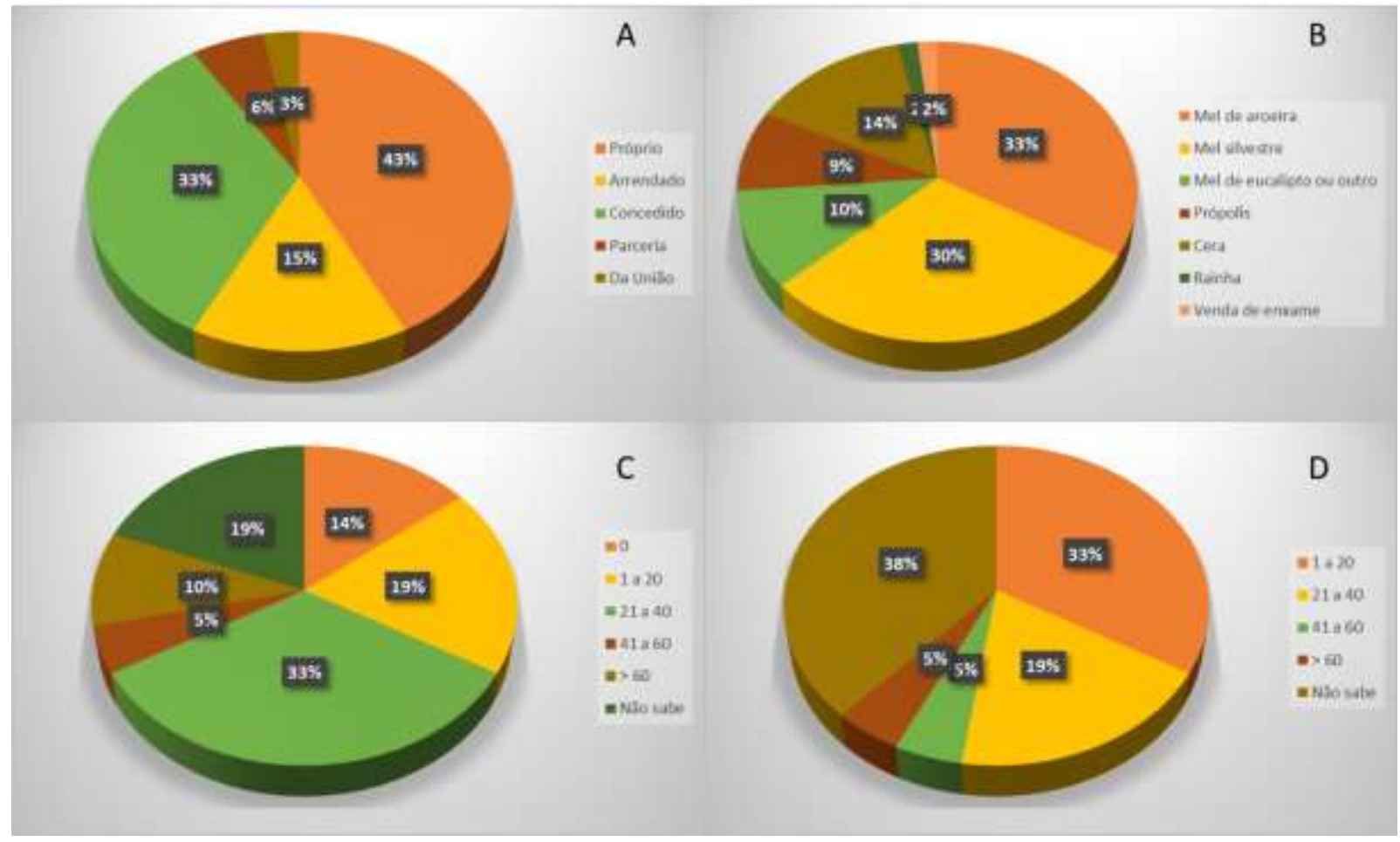

Fonte: Autores (2021).

Um ponto importante a se destacar, é que de fato o interesse dos apicultores, são os produtos oriundos da apicultura, que trata exatamente, dos produtos das abelhas, tais como, mel, pólen, cera, geleia real, própolis e o próprio veneno. Há ainda o trabalho de polinização das abelhas que, para a produção agrícola, tem valor incomparável, do ponto de vista econômico (Klein et al., 2007; Kremen et al., 2007). O produto que apresentou maior destaque na produção apícola do Vale do Jequitinhonha foi o mel, sobressaindo o mel de aroeira seguida pelo mel silvestre (Figura 1B), corroborando com os dados do estudo de Gonçalves et al. (2019). Outros produtos como a própolis, a cera, a venda da rainha ou do enxame somaram 27\% (Figura 1B).

De fato, o mel produzido a partir da floração da Aroeira e silvestre no Vale do Jequitinhonha merece destaque e carece de estudos adicionais. Um estudo realizado na região apresentou que o mel produzido a partir dessas floradas foi capaz de reduzir a proliferação e migração das células do carcinoma epidermoide de boca (CEB), em uma análise realizada in vitro (Sobrinho Santos et al, 2018a). No entanto, os autores afirmam a necessidade de novas investigações para determinar os mecanismos moleculares envolvidos no potencial antiproliferativo e antimigratório do mel (Sobrinho Santos et al, 2018a).

Em relação à área total da propriedade (Figura 1C), observa-se que 33\% dos apicultores entrevistados possuem uma área de terra de até 40 ha, caracterizando-se como propriedades de agricultura familiar com diversificação das atividades. A diversificação agrícola pode ser uma alternativa para pequenos produtores aumentarem seus rendimentos, e por consequência melhorar a qualidade de vida. A diversificação das atividades no campo surge da necessidade de melhorar as condições da agricultura familiar e da grande importância de elaborar estratégias que possibilitem a melhora das condições dos produtores familiares (Barbosa et al., 2016). Nesse sentido, percebe-se que os apicultores entrevistados realizam a atividade de apicultura como uma forma de diversificar a propriedade, obtendo a garantia da fonte de renda para a família.

Essa diversificação das atividades agropecuárias pode ser deduzida a partir das áreas que são utilizadas para a apicultura. Apenas 19\% dos apicultores destinam área de 21 a 40 ha para a atividade apícola. A maioria não sabe definir ao certo a dimensão de área destinada à apicultura, enquanto 33\% dos apicultores utilizam uma área de 1 a 20 ha para a apicultura 
(Figura 1D). A não utilização da área total para uma única atividade agropecuária possibilita a segmentação dos mercados e da diferenciação dos produtos. Além disso, a diversificação da produção pela propriedade constitui uma estratégia que proporciona vantagens competitivas aos negócios agrícolas, com base na economia de escopo. A diversificação da produção é uma estratégia que pode ser utilizada pelos agricultores com o objetivo de enfrentar as adversidades da produção e do mercado (Barbosa et al., 2016).

$\mathrm{Na}$ distribuição de frequência da produtividade entre os apicultores, verificou-se que a produtividade menor que 2 toneladas ocorrem em $62 \%$ dos apicultores e para a produtividade acima de 2 toneladas a ocorrência é de $24 \%$ dos apicultores (Figura 2A). Conforme informado na literatura, o volume da produção brasileira de mel varia conforme a fonte dos dados, em função de se adotar metodologias distintas. De acordo com a Pesquisa Pecuária Municipal - PPM, a produção de mel no Brasil atingiu 41 milhões de $\mathrm{kg}$ em 2017, sendo superior ao ano de 2013, com uma produção de 35 milhões de kg (Nunes \& Heindrickson, 2019).

A produção nacional de mel também se concentra em algumas mesorregiões brasileiras. A produção de mel na região Metropolitana de Belo Horizonte (MG) foi de $843.549 \mathrm{~kg}$, correspondendo a 2,06\% da quantidade de mel produzida no Brasil (Nunes \& Heindrickson, 2019). Portanto, no Vale do Jequitinhonha os apicultores que participaram da pesquisa produzem quantidade aquém da observada em outras regiões do estado mineiro, demonstrando que a região precisa melhorar o desempenho técnico na produção e consequentemente elevar a produtividade de mel no apiário.

Figura 2. Dados sobre produtividade, renda e desenvolvimento da atividade apícola. A - Produção anual média de mel (kg). B - Tempo que pratica a apicultura (anos). C - Quantidade de pessoas na atividade. D - Única fonte de renda?

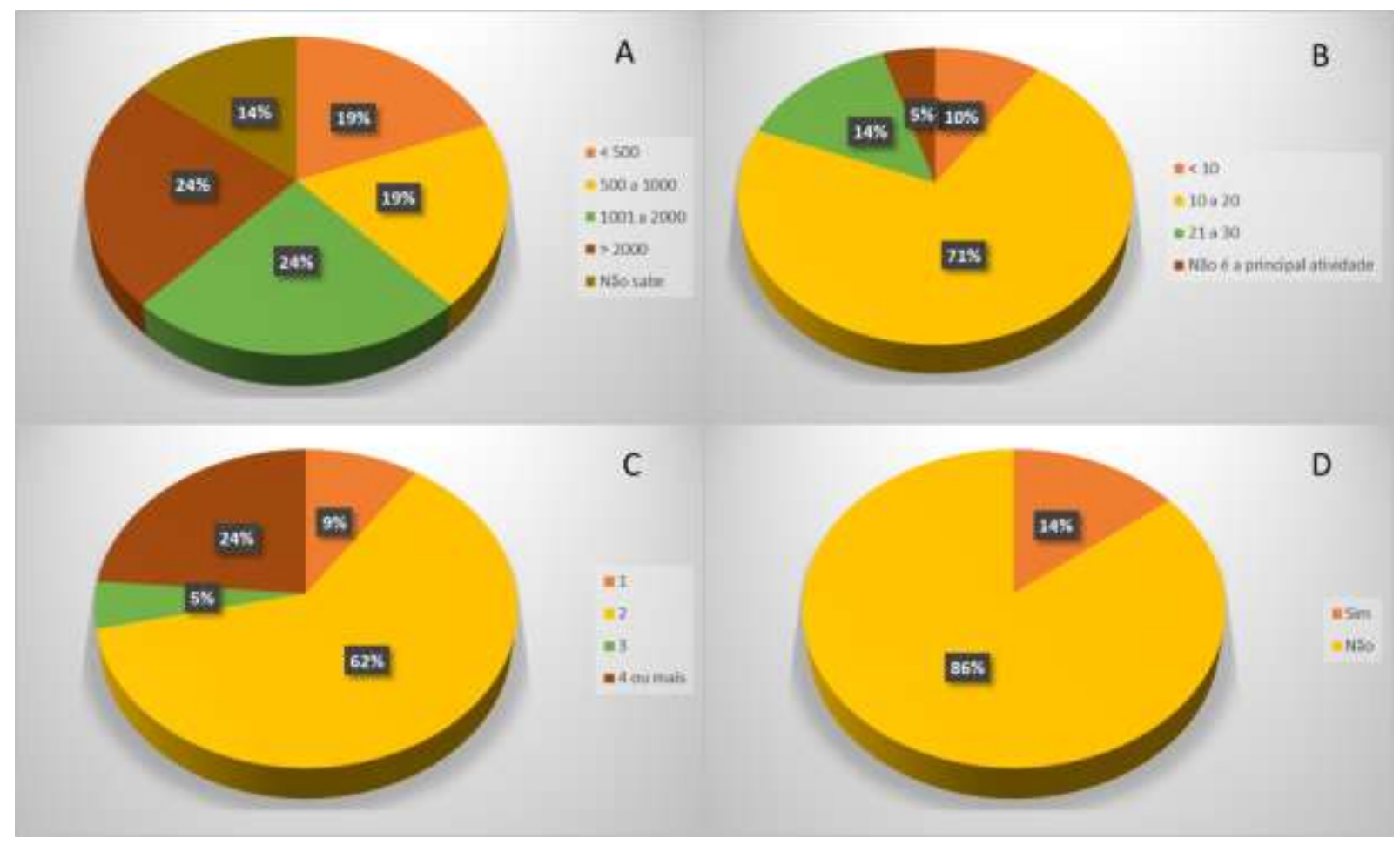

Fonte: Autores (2021).

No âmbito da presente pesquisa, a grande maioria dos apicultores apresenta um tempo de permanência na atividade apícola entre 10 e 20 anos. Apenas $10 \%$ dos apicultores não completaram 10 anos de permanência na atividade e $14 \%$ deles estão na atividade há mais de 20 anos (Figura 2B). Esses dados corroboram com relatos de que os apicultores da região 
possuem experiência no exercício da apicultura e já realizaram algum tipo de curso para auxiliar nas atividades apícolas, e em algum momento tiveram oportunidade de participar de treinamentos relacionados à atividade (Gonçalves et al., 2019).

Portanto, pode-se afirmar que, de modo geral, os apicultores entrevistados apresentam capacidade de desenvolvimento pleno da atividade, pois de acordo com a classificação brasileira de ocupação do Ministério do Trabalho e Emprego o alcance do pleno desempenho da profissão de apicultor demanda o período mínimo de 04 (quatro) anos de experiência (ocupação apicultor código 6134-05 na homepage: www.mtecbo.gov.br). O tempo de permanência na apicultura proporciona ao apicultor a possibilidade de conhecer assuntos relacionados às técnicas de manejo e condução de um apiário, promovendo assim maior experiência e entendimento das relações existentes entre a apicultura e os fatores que a cercam (dos Reis \& Pinheiro, 2011).

Em relação à tipologia de classificação da atividade apícola, de acordo com o número de pessoas envolvidas nesta atividade, pode-se sugerir que a maior parte dos apicultores é predominantemente familiar, pois a região apresenta uma distribuição de frequência em que $76 \%$ de seus apiários empregam até três pessoas na atividade. Já $24 \%$ dos apicultores entrevistados relataram a presença de mais de quatro pessoas trabalhando nos apiários (Figura 2C). No estudo realizado por Gonçalves et al. (2019), 36\% das famílias entrevistadas, há a presença de mais de uma pessoa envolvida com a atividade. Neste referido estudo, a apicultura é praticada utilizando tanto a mão de obra familiar quanto a não familiar, demonstrando que o exercício apícola pode ser praticado por pequenos e grandes produtores, constituindo uma atividade importante e promissora para ambos (Gonçalves et al., 2019).

Estudos demonstram a viabilidade da apicultura como atividade para ocupação da mão de obra familiar, conciliando rentabilidade com pequena quantidade de investimento, permitindo o enquadramento da atividade em programas específicos para o fomento da agricultura familiar (Oliveira et al., 2004; Both, 2006).

Com relação à fonte de renda (Figura 2D), observa-se que $86 \%$ dos entrevistados possuem outras fontes de renda além da apicultura, em contrapartida, para 14\% dos apicultores entrevistados a apicultura é a única fonte de renda. No entanto, no estudo realizado por Gonçalves et al. (2019), verificou-se que a principal fonte de renda das famílias é composta por recursos provenientes da produção e comercialização do mel.

Acredita-se que a busca pelo aumento da renda seja a principal razão para os apicultores da região iniciarem a atividade apícola, independente se esta é realizada em paralelo ou não com outras atividades. Além disso, sugere-se que a presença de produtores rurais no setor ocorre principalmente em virtude do estímulo proporcionado pelo mercado nos últimos anos em relação ao preço do quilo do mel pago ao apicultor (Perez et al., 2005).

A renda proporcionada pela produção de mel na região reforça a análise de vários especialistas sobre a possibilidade da apicultura ser uma alternativa de suplementação de renda para o agricultor familiar (Wolff et al., 2008). Entretanto, é importante observar que a atividade apícola apresenta capacidade de crescimento, justamente pelos potenciais existentes no Vale do Jequitinhonha onde a presença de flora, mercado consumidor regional e incremento no tempo de dedicação do apicultor na condução do negócio podem e devem ser explorados. Conforme demonstrado anteriormente, a maior parte dos apicultores possui outras fontes de renda e somente uma pequena parcela dedica integralmente o seu tempo de trabalho a atividade apícola.

Este cenário revela o potencial a ser desenvolvido e explorado pela atividade, quanto ao grau de importância em relação às demais atividades econômicas exercidas pelos apicultores. É verificado que grande parte dos pastos apícolas existentes na região é pouco explorada. Desta forma, o fato da apicultura para a maior parte dos apicultores ocupar uma posição de atividade marginal, com características de complementaridade, evidencia o quanto a atividade pode crescer e proporcionar maior participação na composição da renda dos produtores rurais do Vale do Jequitinhonha. 
Nesse sentido, é importante traçar estratégias para as possibilidades de maior uso do potencial existente na região, como por exemplo, iniciar negociação de parceria entre empresas de reflorestamento e grupos de apicultores cooperados da região, onde o foco é exatamente viabilizar o aumento da renda dos produtores rurais através do incremento da atividade, explorando a potencialidade presente na flora da região para produzir mel natural em quantidade e com qualidade.

Ações coletivas entre os apicultores poderiam atenuar desafios e acelerar o alcance da solução de problemas como a dificuldade de aquisição de equipamentos e infraestrutura, controle de qualidade dos produtos, comercialização, dentre outros. Este tipo de situação pode ser construída por meio de cooperativas. Iniciativas desta natureza já ocorrem em algumas regiões brasileiras que desenvolvem a apicultura, e os estudos comprovam que estas ações têm proporcionado resultados satisfatórios, promovendo inclusive a mudança da realidade local (Both, 2006; Tschoeke et al., 2006; Veit, 2003).

No Vale do Jequitinhonha, os apicultores estão indo de encontro a esta perspectiva. Dentre os apicultores entrevistados 67\% são cooperados da COOAPIVAJE por pelo menos há 10 anos, enquanto $28 \%$ fazem parte da cooperativa há mais de 10 anos (Figura 3A). A literatura aponta que os apicultores conduzem informalmente a atividade em associações, sugerindo uma necessidade eminente de assistência técnica especializada para o exercício correto do associativismo associado à obtenção de lucros (Gonçalves et al., 2019).

Figura 3. Dados referentes à participação dos apicultores na Cooperativa. A - Há quanto tempo faz parte da COOAPIVAJE? (anos). B - Entrega a produção no entreposto? C - Possui casa do mel?. D - Avaliação do trabalho da cooperativa.

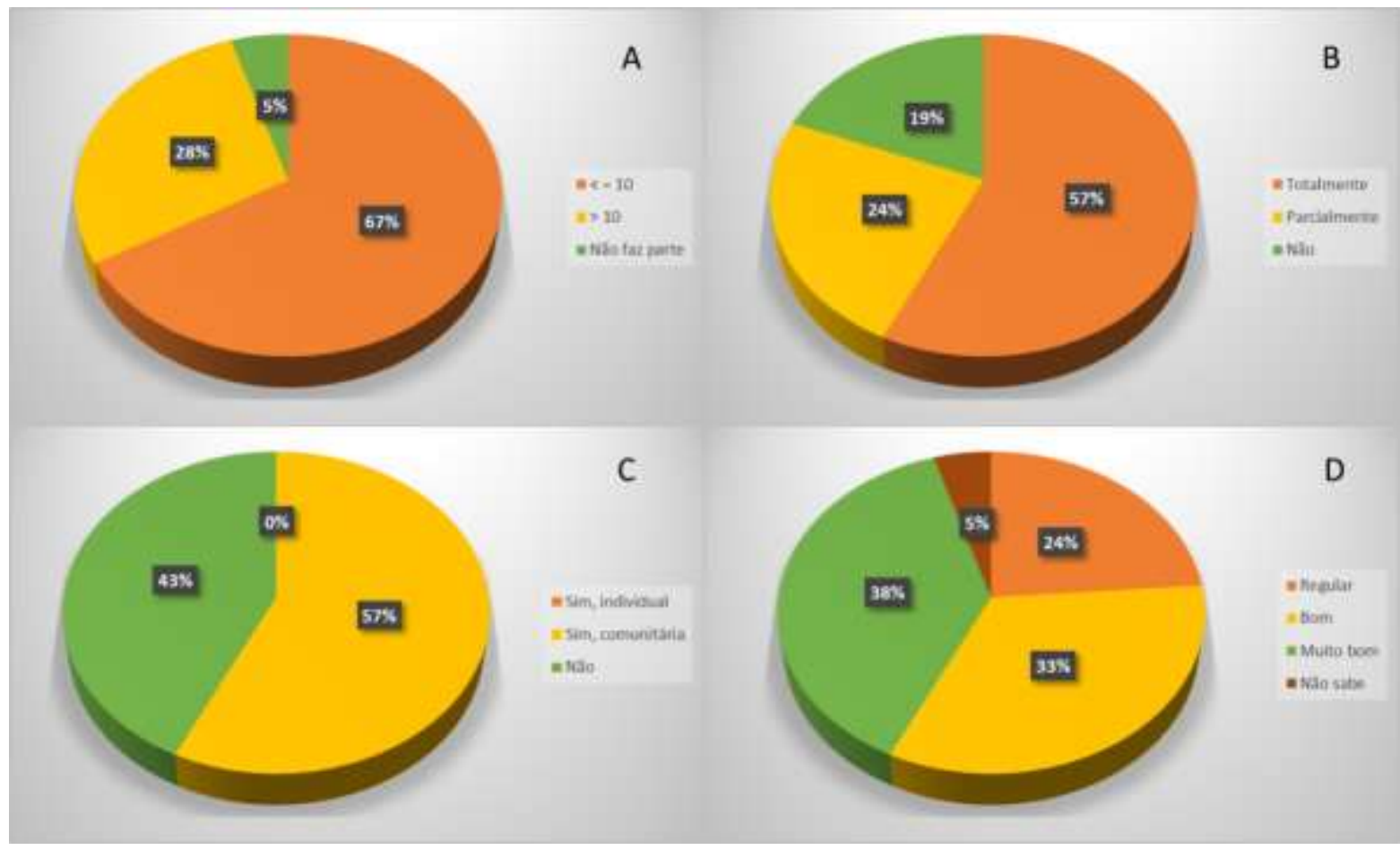

Fonte: Autores (2021).

As cooperativas constituem-se no principal meio para fortalecer os empreendimentos de pequeno porte, onde sua ação confere maior poder de negociação proporcionando economias de escala e de aglomeração (Sachs, 2003; Abromavay et al., 2003; Miranda, 1997). Entretanto, entre os apicultores do Vale do Jequitinhonha essa relação com a cooperativa não tem acontecido de forma efetiva. No que tange a comercialização dos produtos apícolas, foi observada uma frequência de $43 \%$ de apicultores que comercializam total ou parcialmente os seus produtos sem o intermédio da cooperativa (Figura 3B). A 
justificativa para esse fato parece ser principalmente em decorrência do baixo preço pago pela cooperativa, o que não estimula os apicultores a utilizarem a cooperativa como forma de escoamento dos seus produtos.

O uso de equipamentos adequados e a certificação sanitária são práticas tecnológicas fundamentais a serem adotadas no processo produtivo, para a obtenção da qualidade do produto final (Wiese, 1995; Couto e Couto, 1996; Costa et al., 2005; Barreto et al., 2006). Assim, a adoção de boas práticas de higiene, bem como a existência de um local adequado para o manuseio e extração de mel, a chamada casa do mel, são ações prioritárias para o alcance das condições mínimas necessárias exigidas por lei e a consequente competitividade de mercado (Perosa et al., 2004).

No Vale do Jequitinhonha 43\% dos apicultores entrevistados relataram ausência da infraestrutura da casa do mel e os outros 57\% dos entrevistados utilizam uma casa do mel comunitária (Figura 3C). Esta situação revela que grande parte dos apicultores improvisa um local de trabalho para manusear e extrair o mel dos favos, sendo esse local até mesmo uma cozinha de residências. É importante observar que a manipulação dos produtos apícolas deve sempre ocorrer em ambiente apropriado com a infraestrutura adequada. No entanto, no Vale do Jequitinhonha quase metade não possui o local adequado, esta situação revela a necessidade da implantação de um programa que promova o alcance da qualidade do produto, principalmente nas questões relacionadas ao manuseio em local correto.

Verifica-se que, no Vale do Jequitinhonha, a maior parte dos apicultores começa a atividade apícola com uma infraestrutura mínima e a providência para instalação e manuseio em local adequado não ocorre necessariamente no início do desenvolvimento da atividade, visto que a maioria dos apicultores entrevistados exerce a atividade há mais de 10 anos. Acredita-se que a justificativa dos apicultores da região, que não possuem casa do mel, está majoritariamente pautada no impasse da necessidade do alto investimento com a baixa produção de mel existente no apiário. Como a produção é pequena, o apicultor não possuiu estímulo ou recurso financeiro suficiente para implantar a casa do mel.

$\mathrm{O}$ grande esforço e comprometimento dos apicultores com a atividade os fazem alcançar resultados satisfatórios com relação à qualidade do mel, mesmo não possuindo equipamentos e infraestrutura adequados. Estudos indicaram que amostras de mel provenientes do Médio Jequitinhonha atenderam aos padrões de identidade e qualidade da normativa brasileira, embora mais estudos sejam necessários (Sobrinho Santos et al., 2018b).

No trabalho realizado por Gonçalves et al. (2019) foi verificado que a maioria dos entrevistados realiza a retirada do mel das melgueiras na chamada "casa do mel", um local específico para tal finalidade. Entretanto, a "casa do mel" a que se referem os autores é apenas um local destinado ao processamento do mel, sem, contudo, apresentar uma estruturação adequada. Uma vez que, para a extração do mel, nenhum dos entrevistados utiliza centrífugas e mesa desoperculadora. $\mathrm{O}$ autor considera uma vantagem ter esse local específico para uso dos apicultores e relaciona essa vantagem à presença da cooperativa, que oferece aos apicultores associados uma infraestrutura adequada para a coleta do mel.

Desta maneira a produção de mel com higiene e qualidade conforme recomendam os órgãos competentes, deve perpassar por ações coletivas, pois alguns apicultores, que não possuem a infraestrutura adequada, declararam a adoção de estratégias comunitárias, onde o eixo desta ação pode se sustentar na cooperativa, que emprestam o equipamento para a realização dos procedimentos de extração do mel.

Portanto, a atuação da cooperativa junto aos apicultores possui papel relevante na resolução de problemas em diferentes vertentes na apicultura. A garantia do desenvolvimento da atividade apícola depende de uma ação conjunta, estruturada em programas de fomento, baseado em acompanhamento técnico adequado (Souza, 2006). É sabido que a cooperativa tem realizado vários esforços para viabilizar o desenvolvimento da apicultura, entretanto há ainda o que ser feito. A cooperativa deve atuar incentivando os apicultores desde o início de sua criação, fornecendo informações básicas iniciais, e sendo um ponto de apoio a quem os apicultores possam recorrer ao sentirem dificuldades. No estudo realizado por Barbosa e Cardoso (2020) foi apontado que a associação é uma peça chave para o desenvolvimento da atividade e consequentemente da 
região. Na opinião de $71 \%$ dos apicultores entrevistados a atuação da cooperativa é classificada como boa ou muito boa (Figura 3D).

Entende-se que as ações de apoio da cooperativa devem estar além da proposta de transferência de informação e do atendimento da demanda pontual dos apicultores. Dessa forma, as ações devem ocorrer com a existência de um programa específico para a atividade apícola revelando aspectos importantes a serem analisados. O primeiro aspecto é a articulação entre o setor público e privado para promover e consolidar efetivamente o desenvolvimento do arranjo produtivo apícola na região, e o segundo aspecto é a demonstração da preocupação, mesmo que de forma tímida por parte de integrantes do setor apícola da região, em promover iniciativas relacionadas à organização e produção do mel no Vale do Jequitinhonha.

O nível de confiança e a expectativa entre os indivíduos estão relacionados ao capital social e influencia a ação coletiva do grupo (Marteleto \& Silva, 2004). Dessa forma, os valores, a identidade, a instituição e o relacionamento presente no grupo permitirão o desenvolvimento da cooperação e ação de apoio entre os agentes participantes do grupo da apicultura.

Por meio de dados extraídos da literatura, verifica-se que a presença de uma cooperativa contribui para a produção de alimentos de qualidade e com segurança assegurada. Em um estudo que realizou a caracterização qualiquantitativa do mel de Aroeira do Vale do Jequitinhonha, ao avaliar parâmetros macroscópicos, microscópicos, físico-químicos e microbiológicos verificou-se que esse mel, nas amostras avaliadas, atendia, em sua maioria, aos requisitos básicos de controle de qualidade exigidos pelos órgãos oficiais (Sobrinho Santos et al., 2018c) e a atuação de uma cooperativa na região pode ter contribuído para a obtenção de um mel de qualidade.

\section{Considerações Finais}

A região do Vale do Jequitinhonha apresenta indicadores importantes para o fortalecimento do arranjo produtivo do mel, pois a presença de universidades e institutos federais relacionados ao setor, a existência de associações e cooperativas, o estoque de reserva natural através da flora existente na região e o potencial de consumo do produto mel na própria região, permite inferir que estes fatores condicionam uma capacidade de desenvolvimento e evolução da apicultura na região sem precedentes. Além disso, a presença de um contingente considerável de apicultores com tempo de atividade na apicultura superior a 04 anos demonstra a tradição da atividade na região.

Entretanto, percebe-se ao longo deste estudo que estratégias focadas em ações coletivas precisam ser priorizadas para consolidar os elos entre os envolvidos e proporcionar a condição necessária para o fortalecimento da apicultura regional. Nesse sentido, a cooperativa tem um papel importante e, portanto, são necessários esforços para torná-la constante, uma vez que sua ocorrência atualmente não alcança a todos os apicultores. Vislumbra-se, pois, a implantação de projeto ou programa na região, para equacionar a questão da orientação e acesso aos equipamentos adequados em ambientes apropriados conforme preconiza a lei.

É fundamental que o apicultor, de maneira organizada, e o poder público estabeleçam um modelo de desenvolvimento sustentável para a apicultura regional amparado na alta produção de mel com qualidade e consequente consolidação do arranjo produtivo local do mel. Para isto será necessário que o apicultor, através de ações coletivas e o intermédio da cooperativa, produza mel conforme as normas técnicas recomendadas e os agentes fiscalizadores da vigilância sanitária atuem com maior rigor nos canais de distribuição, coibindo veemente o comércio ilegal de produtos adulterados, falsificados e não certificados. E dessa forma, contribuindo para o estabelecimento da Identificação Geográfica do mel no Vale do Jequitinhonha.

\section{Agradecimentos}

Os autores agradecem ao Instituto Federal do Norte de Minas Gerais (IFNMG), Campus Araçuaí; à Universidade Federal dos Vales do Jequitinhonha e Mucuri (UFVJM), Campus Diamantina; à Cooperativa de Apicultores do Vale do 
Jequitinhonha (COOAPIVAJE); ao Conselho Nacional de Desenvolvimento Científico e Tecnológico (CNPq) pelo apoio na realização desta pesquisa.

\section{Referências}

Abramovay, R. (2003). Mercados do empreendedorismo de pequeno porte no Brasil. En: Pobreza e mercados no Brasil: uma análise de iniciativas de políticas públicas-Brasília: CEPAL/DFID, 2003-p. 233-311.

Barbosa, P. J. F., Mendonça, J. C. A., Casarotto, E. L., Machado, R. R., de Almeida, V. L., \& Vitorino Filho, V. A. (2016). A importância da diversificação agrícola como complemento na renda familiar na região de Manhuaçu-MG. Revista do CCEI, 20(35), 1-11.

Barbosa, S. L., \& Cardoso, P. H. G. (2020). Beekeeping Activity Developed by the Beekeepers Association in Cariús-CE. Research, Society and Development, 9(7), e932974913. https://doi.org/10.33448/rsd-v9i7.4913

Barreto, L., Peão, G. F. R., \& Dib, A. D. S. (2006). Higienização e sanitização na produção apícola. Taubaté: Cabral Editora.

Both, J. (2007). Produção de mel de abelhas Apis mellifera L.: a atividade apícola como alternativa de renda em unidade de produção familiar, no município de Castanhal, estado do Pará. Belém: Universidade Federal do Pará.

Boyd, H., \& Westfall, R. (1982). Pesquisa mercadológica e casos. FGV (Serviços de Publicações).

Campomar, M. C. (1981). As atividades de marketing no processo de transferencia de tecnologia:(um estudo sobre instituições de pesquisa governamentais).

Costa, C. C. D., Pereira, R. G., \& Prata Filho, D. D. A. (2005). Influência de centrífuga no processamento do mel de abelha. Engenharia Agrícola, 25, 809816.

Couto, R. H. N. \& Couto, L. A. (1996). Apicultura: manejos e produtos. FUNEP.

de Oliveira, F., Costa, S. M. A. L., Tarsitano, M. A. A., \& Sant'Ana, A. L. (2004). Produção de mel na região noroeste do Estado de São Paulo: um estudo de caso de produtor familiar.

de Oliveira, M. C. C. (2018). A cooperativa agrícola na reorganização produtiva do território: a experiência da Central de Cooperativas Apícolas do Semiárido Brasileiro. Revista Cadernos de Ciências Sociais da UFRPE, 1(12), 137-155.

dos Reis, V. D. A., \& Pinheiro, R. D. S. (2011). Fundamentos para o desenvolvimento seguro da apicultura com abelhas africanizadas. Embrapa PantanalDocumentos (INFOTECA-E).

Fávero, C., \& Monteiro, F. T. (2014). Disputas territoriais no Vale do Jequitinhonha: uma leitura pelas transformações nas paisagens. Revista Agriculturas, 11(3), 07-15.

Food And Agriculture Organization - FAO. (2018). Production. http//faostat.fao.org.

Gonçalves, J. R. S. M., Santos, E. M. S., Santos, H. O., Costa, I. C., Paixão, D. M., Alves, J. N., \& de Souza Costa, K. (2019). Aspectos da apicultura: entrevistas com apicultores da Cooperativa do Vale do Jequitinhonha. Caderno de Ciências Agrárias, 11, 1-10.

IBGE (2010). Censo Demográfico. http://www.sidra.ibge.gov.br/.

IBGE (2015). Divisão políticoadministrativa do Brasil. http://www.sidra.ibge.gov.br/.

Klein, A. M., Vaissiere, B. E., Cane, J. H., Steffan-Dewenter, I., Cunningham, S. A., Kremen, C., \& Tscharntke, T. (2007). Importance of pollinators in changing landscapes for world crops. Proceedings of the royal society B: biological sciences, 274(1608), 303-313.

Kremen, C., Williams, N. M., Aizen, M. A., Gemmill-Herren, B., LeBuhn, G., Minckley, R., \& Ricketts, T. H. (2007). Pollination and other ecosystem services produced by mobile organisms: a conceptual framework for the effects of land-use change. Ecology letters, 10(4), 299-314.

Lengler, L., Lago, A., \& Coronel, D. A. (2007). A organização associativa no setor apícola: contribuições e potencialidades. Organizações Rurais \& Agroindustriais, 9(2), 151-163.

Marteleto, R. M., \& Silva, A. B. D. O. (2004). Redes e capital social: o enfoque da informação para o desenvolvimento local. Ciência da informação, 33, 4149.

Minas Gerais, (2020). Cartilha Circuito Mineiro de Oportunidade e Negócios. http://www.desenvolvimento.mg.gov.br/assets/projetos/1065/cd1cd8c6644d3 2afe5ea9d6bdcfe3520.pdf.

Miranda, D. (1998). Associativismo rural, agroindústria e intervenção: estudo de caso em uma associação de produtores familiares. Universidade Federal de Lavras-MG.

Neto, A. F., Monteiro, A. I. S., Silva, J. C. S, \& Souza, M. M. A. (2020). Desenvolvimento do Semiárido: Organizações, Gestão, Inovação e Empreendedorismo. Petrolina: Univasf, 155p.

Nunes, S. P., \& Heindrickson, M. (2019). A cadeia produtiva do mel no Brasil: análise a partir do sudoeste Paranaense. Brazilian Journal of Development, 5(9), 16950-16967.

Perez, L. H., Resende, J. V. de Freitas, B. B. de. (2005). Mel: exportações fazem produção aumentar de Norte a Sul. 
Research, Society and Development, v. 10, n. 10, e295101018891, 2021

(CC BY 4.0) | ISSN 2525-3409 | DOI: htttp://dx.doi.org/10.33448/rsd-v10i10.18891

Perosa, J. M. Y., Arauco, E. M. R., Santos, M. D. A., \& Albarracín, V. N. (2004). Parâmetros de competitividade do mel brasileiro. Inf. Econ, $34,41-48$.

Sachs, I. (2003). Inclusão social pelo trabalho: desenvolvimento humano, trabalho decente e o futuro dos empreendedores de pequeno porte. Editora Garamond.

Sachs, I. (2009). Caminhos para o desenvolvimento sustentável. (2002). Garamond.

Santos, D. L. D. R. D. (2013). Processo de diversificação na agricultura familiar no município de Arvorezinha-RS.

Santos, E. M. S., Santos, H. E. O. I., Gonçalves, J. R. S. A. M., Almeida, A. C., Brandi, I. V., Cangussu, A. R., \& Costa, K. S. (2018). Quali-quantitative characterization of the honey from Myracrodruon urundeuva allemo (Anacardiceae-Aroeira): macroscopic, microscopic, physico-chemical and microbiological parameters. African Journal of Biotechnology, 17(51), 1422-1435.

Santos, E. M. S., Santos, H. O., Brandi, I. V., Santos, G. L. M., de Jesus Viana, M. I., Araújo, B. R. S., ... \& Neiva, R. J. (2018). Caracterização do mel do Médio Jequitinhonha-Brasil: uma abordagem preliminar/Characterization of the honey of the Jequitinhonha Valley-Brazil: a preliminary approach. Caderno de Ciências Agrárias, 10(1), 45-51.

Santos, E. M. S, Santos, H, O., Gonçalves, J. R., Sá M., Almeida, A. C., Brandi, I. V., Cangussu, A. S. R., Alves, J. N., de Jesus, S. F., Neiva, R. J., Costa, K. de S., Guimarães, A. L. S., \& Farias, L. C (2018). Atividade do mel na proliferação e migração do carcinoma epidermóide oral: análise in vitro e bioinformática. Scientific Research and Essays, 13 (15), 158-171.

Souza, D. C. (2006). ADRs-Os agentes da nova apicultura no Brasil. Revista SEBRAE Agronegócios, 3, 46-47.

Tschoeke, P. H.; Bessa, J. C. A.; Ruiz, F. F. E \& Silveira, M. C. A. C. (2006). Aspectos relacionados à produção e manejo apícola na região sul do Tocantins. In: XVI Congresso Brasileiro De Apicultura.

Wiese, H. (1995). Novo manual de apicultura.

Wolff, L. F., dos Reis, V. D. A., \& dos Santos, R. S. S. (2008). Abelhas melíferas: bioindicadores de qualidade ambiental e de sustentabilidade da agricultura familiar de base ecológica. Embrapa Clima Temperado-Documentos (INFOTECA-E). 